\title{
From CERN knowledge to society
}

\author{
Manuela Cirilli ${ }^{1}$ \\ CERN \\ CH 1211 Geneva, Switzerland \\ E-mail: Manuela.Cirilli@cern.ch
}

CERN's mission is the pursuit of knowledge through curiosity-driven research. The Laboratory was created to explore the fundamental particles and their interactions, with the goal of helping us to understand how the universe works. Since its beginnings, CERN has also acted as a trailblazer in the technologies related to accelerators, detectors and computing. As a laboratory with a long-term research plan, it is continuously innovating. For more than 60 years, the work of thousands of scientists from all over the world has pushed back the limits of knowledge in fundamental physics, as well as in many fields of technology. It is part of CERN's mission to ensure that our innovations bring practical benefits to society as a whole.

We use a variety of avenues to bring CERN innovation to society, ranging from education, communication and outreach to formal Knowledge Transfer (KT) activities whose aim is to maximise the positive impact of CERN technologies outside the HEP field. CERN's KT activities have led to hundreds of collaboration agreements from the field of MedTech and aerospace, and from industry 4.0 to cultural heritage. We have a CERN KT fund to stimulate innovation. It has funded 41 projects since 2011, while our Medical Applications budget has funded 25 projects since 2014. We promote entrepreneurship and we grant licences for companies to develop our technologies. 23 start-ups are now using CERN technology, some hosted in the nine Member State Business Incubation Centres (BICs). All this is done to ensure a strong return on investment for policy makers, industry, and the general public.

The 39th International Conference on High Energy Physics (ICHEP2018)

4-11 July, 2018

Seoul, Korea

\section{${ }^{1}$ Speaker}




\section{Introduction}

CERN is the world's largest particle-physics laboratory, located at the border between France and Switzerland. Its core mission is fundamental research in particle physics. Yet, as a publicly funded laboratory, it also has a remit to ensure that its technology and expertise deliver prompt and tangible benefits to society wherever possible. Other physics-research laboratories and institutes were early adopters of CERN technologies, thanks to the highly collaborative nature of particle physics. Since its creation in 1954, CERN has also been active in transferring technology to industry, mainly through purchasing contracts or collaboration agreements. Through novel developments in the field of accelerator technologies and detectors, and more recently in computing and digital sciences, CERN technologies and know-how have contributed to applications in many fields, including the World Wide Web, invented at CERN by Tim BernersLee in 1989. This paper illustrates some concrete cases of CERN technologies and know-how applied to diverse fields, and describes how CERN's Knowledge Transfer group supports the Laboratory's knowledge- and technology-transfer activities.

\section{From CERN technologies to a wide variety of application areas}

CERN's expertise builds broadly on three technical fields: accelerators, detectors and computing. Behind these three pillars of technology, lies a great number of areas of expertise: from cryogenics to ultra-high vacuums, from particle tracking to superconductivity and many more. These technologies, and the human expertise associated with them, translate into positive impact on society in a wide range of fields, from medical and biomedical technologies to aerospace applications, safety, "industry 4.0", cultural heritage and emerging technologies.

\subsection{Technologies for health}

At CERN, early activities with pertinence to medical applications date back to the 1970 s, and have been triggered for the most part, by individual initiatives in the fields of Positron Emission Tomography (PET), X-ray and gamma-ray imaging. In the 90's, CERN witnessed the first collaborative endeavours in medical applications, with the establishment of the Crystal Clear and Medipix collaborations, and the partnership with TERA Foundation, MedAustron, and Onkologie 2000 to initiate the Proton Ion Medical Machine Study (PIMMS).

Since 1997, CERN's Crystal Clear collaboration (CCC) has been using its expertise in scintillators to develop and construct PET prototypes. Their first success was the ClearPET concept: the development of several prototypes has resulted in the commercialisation of a smallanimal scanner with breakthrough performance, and led to the first simultaneous PET/CT (Computed Tomography) image of a mouse in 2015. Recent CCC developments are focused on time-of-flight PET scanners, ultrafast scintillators and organ-specific imaging.

Medipix is a family of read-out chips for particle imaging and detection developed by the Medipix Collaborations, initially to address the needs of particle tracking at the CERN LHC. The Medipix chips work like a camera, detecting and counting each individual particle hitting the pixels when its electronic shutter is open. This enables high-resolution, high-contrast, noise hit free images - making it unique for imaging applications. A member of the Medipix 3 collaboration founded a company and obtained a licence to exploit the chip for spectral computed tomography 
imaging - X-Ray imaging in colour. In summer 2018, the company developed a scanner based on the Medipix3 technology and managed to take the first 3D colour X-ray images of a human body.

The PIMMS study was carried out between 1996 and 2000, and resulted in a synchrotron design optimised for treating cancer with protons and carbon ions; the design was further adapted by TERA, and finally evolved into the machine built for the CNAO treatment centre in Italy, with seminal contributions from INFN. Later on, MedAustron in Austria built its treatment centre starting from the CNAO design. Beyond the initial design study, CERN contributed to the CNAO and MedAustron treatment centres, in particular with expertise in accelerators and magnets and with training of personnel.

In recent years, teams at CERN have manufactured an innovative radio-frequency quadrupole (RFQ) designed to be compact, modular, low-cost and suitable for medical applications. This high-frequency RFQ capitalises on the skills and know-how developed at CERN while designing Linac 4, and is a perfect injector for the new generation of compact linear accelerators being developed for hadron therapy. Expertise in high-gradient accelerating structures gathered by the Compact Linear Collider (CLIC) group at CERN is also being applied to novel designs for hadron-therapy facilities, as well as to the development of accelerators to boost the energy of medical cyclotrons to provide proton-imaging capabilities. Accelerator technologies in which CERN is at the forefront include superconducting magnets: leveraging on this expertise, CERN has proposed a new, innovative gantry design based on a toroidal magnet concept, which bends the treatment beam without the need to rotate the structure. Due to the use of superconductors, this gantry will substantially reduce weight and footprint compared to conventional fully rotating gantries, especially for ion beams.

Accelerators are also increasingly needed for the production of radioisotopes, which are used in nuclear medicine imaging and therapy. Artificially-made radioisotopes are still mostly produced by research reactors, but new accelerator-based technologies are being explored for next-generation facilities that would replace the wobbly reactors. The ISOLDE accelerator facility at CERN has constantly been developing the so-called Isotope Separation Online (ISOL) technique, which is now being taken one step further by the CERN-MEDICIS installation, dedicated to the production of a wide range of innovative radioisotopes for medical research.

Simulation codes initially developed for HEP, such as Geant4 and FLUKA, have also become crucial to modelling the effects of radiation on biological tissues for a variety of applications in the medical field. FLUKA, jointly developed by CERN and INFN, is licensed to various MedTech companies, and Geant4 is adopted by thousands of users worldwide for applications in a variety of domains. Computing tools, infrastructures and services developed at CERN have also great potential for applications in the medical field. CERN openlab has started two collaborative projects in this domain: BioDynaMo aims to design and build a cloud-based computing platform for rapid simulation of biological tissue dynamics, such as brain development; GeneROOT aims to use ROOT to analyse large genomics data sets, beginning with data from TwinsUK, the largest UK adult twins registry.

\subsection{Aerospace}

Aerospace is a field in which CERN is playing a critical and increasingly recognised role, based on many natural synergies. Space missions, accelerator and detector infrastructures alike deal with extreme environments, posing stringent technological requirements that often overlap. 
CERN operates testing facilities and develops qualification technologies for high-energy physics, which are useful for ground testing and qualification of flight equipment. This opportunity is particularly attractive for miniaturised satellites called CubeSats that typically use commercial off-the-shelf components for their electronics, since radiation qualification according to standard procedures is expensive and time-consuming. The CERN Latchup Experiment STudent sAtellite (CELESTA) intends to develop a CubeSat version of RadMon, a radiation monitor developed at CERN, and to prove that low-Earth orbit qualification can be performed in CERN's High energy AcceleRator Mixed field facility (CHARM). CELESTA is being developed in collaboration with the University of Montpellier and in 2017 was selected by ESA's "Fly Your Satellite!" programme.

Magnesium diboride (MgB2), the high-temperature superconductor that will be used for the innovative electrical transmission lines of the high-luminosity LHC, has also demonstrated its potential for future space missions. Within the framework of the European Space Radiation Superconducting Shield (SR2S) project, which aims to demonstrate the feasibility of using superconducting magnetic shielding technology to protect astronauts from cosmic radiation, CERN successfully tested a prototype racetrack coil wound with a $\mathrm{MgB} 2$ superconducting tape.

Astronauts' exposure to space radiation is a major concern for future crewed missions to Mars and beyond. The already mentioned Monte Carlo codes FLUKA and Geant 4 have been routinely used to study the radiation environment of past, recent, and future space missions. The TimePix detectors, which are USB-powered particle trackers based on the Medipix technology, are already used by NASA on board the International Space Station to accurately monitor radiation doses.

CERN's computing expertise is also finding applications in aerospace. To solve the challenge of sharing software and codes in big-data environments, researchers at CERN have developed a system called CernVM-FS (CERN Virtual Machine File Systems), which is now also being used for Euclid, a European space mission that aims to study the nature of dark matter and dark energy, to deploy software in Euclid's nine science data centres.

\subsection{From safety to cultural heritage and beyond}

CERN's unique working environment, which combines various types of radiation, extremely low temperatures, ultra-high magnetic fields and very high voltages, requires innovative solutions for detecting threats and preventing risks. An example is B-rad, a portable meter to ensure radiation safety in strong magnetic fields that was initially developed by CERN's radiation-protection group and fire brigade. With a financial contribution from the CERN Knowledge Transfer (KT) Fund, the product has been brought from lab prototype to finalised product in collaboration with an Italian company. Another example is Kryolize, a novel cryogenic safety software also supported by the CERN KT Fund. Six Kryolize licences have now been granted to other research laboratories, with potential application domains ranging from the food industry to cryogenic techniques in medicine.

The trend toward compact accelerators and the development of pixel detector readout chips is also benefitting the world of cultural heritage applications. The high-frequency RFQ mentioned in section 2.1 is expected to open up new possibilities for the analysis of fine art and cultural artefacts. It will employ a technique called Particle Induced X-ray Emission (PIXE) to measure the elements present within a sample exposed to a beam of low-energy particles. This will enable 
on-site studies of artworks. The project, named MACHINA (Movable Accelerator for Cultural Heritage In-situ Non-desctructive Analysis), is a collaboration between the Italian Istituto Nazionale di Fisica Nucleare (INFN) and CERN, through INFN's cultural heritage network CHNet. The Opificio Delle Pietre Dure, one of the world's leading institutes in the field of art restoration, will be the first user. The Medipix technology developed by the Medipix 2 and Medipix3 collaborations also represents a new tool within the portfolio of non-destructive inspection methods used in the field of art studies. This technology enables X-ray spectral imaging (XRSI), material resolved X-ray imaging and soon X-ray "colour" imaging (XRCI) useful for the inspection of paintings; it has been commercialised by a start-up company based in Prague.

\section{Knowledge Transfer tools}

CERN is an international hub for scientists, engineers and professionals, committed to advancing their fields of expertise. The knowledge created by CERN's community has the potential to create impact by leading to innovation in fields beyond particle physics. This innovation can happen organically, but actively investing in the process can boost its impact and reach. The CERN Knowledge Transfer (KT) group provides advice, support, training, networks and infrastructure to ease the transfer of CERN's know-how to industry and eventually society.

Intellectual property (IP) lies at the core of successful knowledge transfer at CERN. It enables CERN to claim being at the origin of a novel technology, making it possible to share its knowledge to reach society. CERN's policy is to disseminate its technologies as widely as possible to industrial and institutional partners within its Member States, however patenting represents only a tiny part of CERN's approach to IP. CERN will only consider patenting where it might help mitigate the financial risks of investing further in the development of a technology. CERN's patent portfolio is a reflection of this, and currently comprises 34 patent families, a number significantly lower than organisations of a similar size. In addition to its technology portfolio, CERN also has a wealth of scientific and technical competence, across a large variety of expertise, which is accessible through collaboration and consultancy agreements.

At CERN, Open Innovation has been part of our DNA since its inception. Several CERN software technologies are developed with open collaboration in mind, and CERN is also contributing to many open source projects, small or large, that promote collaboration within the community in the larger sense, not only the scientific world. The CERN Open Hardware Licence, drafted and published by the CERN KT group, was born out of the wish to openly disseminate CERN's hardware designs. The licence fosters the dissemination of schematics and hardware documentation, and of improvements made to the hardware. The licence itself can be used by anyone on their own hardware documentation, and is a good example of how addressing CERN's needs can have surprising benefits for society as it encourages the availability of open hardware worldwide.

One of the main challenges in the knowledge-transfer sphere is to make it as easy as possible for scientists and other specialists to turn their research into innovations, and CERN invests much effort in such activities. Launched in 2011, the CERN KT Fund bridges the gap between research and industry by awarding grants to projects proposed by CERN personnel where there is high potential for positive impact on society. Since its creation, 40 projects have been funded, each receiving grants with a value between 15 and $240 \mathrm{kCHF}$ over a period of one or several years. In 2016, two European Commission funded projects, AIDA-2020 and ARIES, incorporated a proof- 
of-concept fund modelled on CERN's KT Fund. CERN also provides a limited amount of seed funding for projects aimed at transferring its technologies and know-how to the medical field. Between 2014 and 2017, 25 projects have been funded with an average grant of about $64 \mathrm{kCHF}$ per project.

Since the early days of technology transfer at CERN, one of the main focuses has been on knowledge transfer through people, especially early career scientists who work in industry after their contracts at CERN or who start their own company. Over the last 20 years, CERN has continued to build a general culture of entrepreneurship within the Organization through many different avenues. There are currently more than 20 start-ups and spin-offs using CERN technologies in their business. To assist entrepreneurs and small technology businesses in taking CERN technologies and expertise to the market, CERN has also established a network of ten Business Incubation Centres (BICs) throughout its Member States where companies can directly express their interest in adopting a CERN technology. The BIC managers provide office space, expertise, business support, access to local and national networks and support in accessing funding. Every year since 2008, students from the School of Entrepreneurship (NSE) at the Norwegian University of Science and Technology (NTNU) spend a week at CERN to evaluate the business commercial potential of CERN technologies. Three of the students attending the CERN-NTNU screening week in 2012 started a spin-off based on CERN's open-source software Invenio. The company has now, among others, contracts to host Invenio for the UNESCO International Bureau of Education, the California Institute of Technology and the Max Planck institute for Extraterrestrial Physics.

Getting the next generation of scientists into the habit of thinking about their research in terms of impact is vital for knowledge transfer to thrive. In 2015, CERN launched a series of Entrepreneurship Meet-Ups (EM-Us) to foster entrepreneurship within the CERN community. Selected CERN and external entrepreneurship experts present their expertise at informal gettogethers and the events offer a good opportunity to network. The CERN \& Society Foundation launched the CERN Entrepreneurship Student Programme (CESP), bringing together graduate students from all around the globe for a 5-week practical and theoretical training at CERN in the summer. The first edition was held in summer 2018, and hosted 10 students. Still in 2018, CERN organised the CERN Medical Technology Hackathon (CERN MedTechHack) in order to explore new ways of developing viable applications of CERN technologies in the medical field. The CERN MedTechHack took place over 3 days, during which international teams of students competed to solve topical problems in the medical field pitched by healthcare organisations and industry partners.

\section{Conclusions}

Knowledge transfer is an integral part of CERN's mission. CERN technologies, infrastructures, and know-how are having a positive impact in a variety of fields beyond particle physics. This mission is supported by a dedicated Knowledge Transfer group, with a broad diversity of tools and avenues being explored to maximise the dissemination of CERN knowledge, and more initiatives will be started to meet the needs of industrial and research partners in CERN's Member States and associate Member States for the coming years. 